\section{Sistema de informação para integrar os dados da assistência oncológica ambulatorial do Sistema Único de Saúde}

\author{
An information system to integrate outpatient \\ cancer care data in the Unified National Health \\ System
}

1 Centro de Ciências Biomédicas, Centro Universitário Serra dos Órgãos, Teresópolis, Brasil. 2 Faculdade de Ciências Médicas, Universidade do Estado do Rio de Janeiro, Rio de Janeiro, Brasil.

3 Instituto Alberto Luiz Coimbra de Pós-graduação e Pesquisa de Engenharia, Universidade Federal do Rio de Janeiro, Rio de Janeiro, Brasil.

Correspondência R. C. Souza Centro de Ciências Biomédicas, Centro Universitário Serra dos Órgãos.

Av. Alberto Torres 111, Teresópolis, RJ 25964-002, Brasil.

romulocsouza@gmail.com

\begin{abstract}
This study focuses on the development of a system to integrate monthly outpatient cancer care data in the Unified National Health System (SUS). The system was modeled to retrace the treatment evolution in each cancer case and services output, with the following advantage: the system focuses on the cancer case, does not require knowledge by the user in order to handle the database, and allows updating the base as new data emerge. The results of direct queries in the system were identical to those obtained from direct inspection of the original database and those from another integration approach. The use of a tool with these characteristics by public administrators can help improve the quality of outpatient cancer care provided by the National Health System.
\end{abstract}

Ambulatory Care Information Systems; Ambulatory Care; Neoplasms
Rômulo Cristovão de Souza 1,2 Sergio Miranda Freire 2

Rosimary Terezinha de Almeida ${ }^{3}$

\section{Introdução}

Os diversos sistemas de informação em saúde implantados no país nas últimas décadas pelo Ministério da Saúde possibilitam a realização de vigilância epidemiológica e de estudos sobre a utilização de serviços.

Um sistema de vigilância é um componente fundamental para o planejamento e acompanhamento do desempenho dos programas de controle do câncer, fornecendo informações sobre a magnitude da doença e do efeito das medidas de prevenção, detecção precoce, tratamento e cuidados paliativos. Atualmente, parte dessas informações está disponível de forma fragmentada em alguns sistemas ou registros de dados.

Os Registros de Câncer de Base Populacional (RCBP) são a base para estimar a incidência das neoplasias, bem como a mortalidade e a sobrevida dos pacientes 1 . Os Registros Hospitalares de Câncer (RHC) fornecem informações sobre a doença, as indicações de tratamento e a evolução dos casos, com base em dados registrados pelos hospitais. Outra fonte de dados sobre a assistência oncológica é o módulo de oncologia do subsistema de Procedimentos de Alta Complexidade do Sistema de Informações Ambulatoriais do Sistema Único de Saúde (APACSIA/SUS). Esse módulo foi criado em novembro de 1998, com a publicação da Portaria GM/MS no . 3.536, de setembro de 1998 2. Uma importante característica do módulo de oncologia do APACSIA/SUS é que 
os seus registros, além de serem de abrangência nacional, são de livre acesso.

Os dados mantidos pelo módulo de oncologia do APACSIA/SUS são obtidos por meio da Autorização de Procedimento de Alta Complexidade (APAC), que é uma autorização prévia necessária para a realização de procedimentos de quimioterapia e radioterapia pelos serviços de saúde credenciados pelo SUS. Embora sua função principal seja a administrativa, voltada para o processo de autorização dos procedimentos, sua implantação não se deu apenas com este propósito. Em seus registros, é possível obter dados sobre os pacientes, os procedimentos de radioterapia e quimioterapia utilizados e os serviços de saúde. Assim, sua concepção buscou criar um instrumento para auxiliar os gestores no planejamento, controle e auditoria dos serviços, além de fornecer dados epidemiológicos para produção científica e avaliação de resultados terapêuticos 3 . Diversos autores têm utilizado este módulo para analisar o perfil dos pacientes com câncer assistidos no sistema público de saúde $4,5,6,7$ e o dimensionamento da infra-estrutura assistencial ${ }^{8}$.

Considerando que a produção dos serviços é armazenada em arquivos mensais distintos, a utilização destes para avaliar a assistência oncológica requer um processo de integração dos arquivos mantidos pelo sistema. Nesse sentido, Gomes Jr. et al. ${ }^{9}$ apresentaram uma proposta visando a integrar esses arquivos em uma única tabela, permitindo, dessa forma, a análise da produção dos serviços e também a identificação dos casos de câncer, descrevendo a assistência a estes. Uma limitação da estratégia proposta é que nem sempre os profissionais de saúde e gestores têm o conhecimento de linguagem de banco de dados necessário para a sua utilização.

Sendo assim, o presente trabalho teve por objetivo o desenvolvimento de um sistema de informação, denominado SISONCO, que permitisse a integração dos arquivos que compõem o módulo de oncologia do APACSIA/SUS de forma automática e oferecesse uma interface amigável para a utilização desses dados.

\section{Materiais e métodos}

Neste trabalho, foram utilizados os arquivos relativos à produção mensal do Estado do Rio de Janeiro do módulo de Oncologia, do APACSIA/ SUS, para o período de janeiro de 2001 a dezembro de 2002, obtidos em novembro de 2007 no site do Departamento de Informática do SUS (DATASUS. ftp://msbbs.datasus.gov.br/Arqui vos_Publicos/Estado_RJ/00_index.htm). Esses arquivos são disponibilizados de forma compactada, sendo identificados como ACUFAAMM, no qual UF, AA e MM significam, respectivamente, unidade federativa, ano e mês de apresentação. Cada arquivo contém dez tabelas de dados, quatro das quais foram utilizadas neste trabalho e são descritas de forma resumida abaixo:

- Produção dos procedimentos de alta complexidade, com 20 campos relativos aos processos administrativos envolvidos para a emissão da APAC, como, por exemplo: código do serviço de saúde, data de referência, código do procedimento e motivo de cobrança.

- Cobrança dos procedimentos de alta complexidade, com 14 campos relativos ao faturamento dos procedimentos apresentados, como, por exemplo, o valor do procedimento.

- Produção de quimioterapia, com 21 campos relativos aos dados dos pacientes em tratamento quimioterápico, como, por exemplo: data de nascimento, gênero, diagnóstico principal, estádio do tumor e meses previstos de tratamento.

- Produção de radioterapia, com 25 campos relativos aos dados dos pacientes em tratamento de radioterapia, como, por exemplo: data de nascimento, gênero, diagnóstico principal, estádio do tumor e número de campos irradiados.

A estrutura de dados do SISONCO foi modelada segundo o diagrama de Entidade-Relacionamentos 10, sendo utilizado o Postgresql versão 8.14 (Postgresql Global Development Group, http://www.postgresql.org) como gerenciador de banco de dados. Utilizou-se a UML (Unified Modeling Language) 11 para a modelagem do sistema, o qual foi implementado em Java versão 6 (Sun MicroSystems, http://www.java.com/pt $\mathrm{br/download/index.jsp).} \mathrm{Uma} \mathrm{interface} \mathrm{gráfica}$ permite ao usuário a atualização e a consulta aos dados do sistema.

O SISONCO permite a realização de consultas relacionadas ao caso de câncer ou à produção do serviço. Adotou-se a definição de caso de Gomes Jr. \& Almeida 12, segundo a qual um caso é identificado por uma chave composta pelos campos: CPF (cadastro da pessoa física), gênero, data de nascimento e o código relativo ao tipo de câncer primário, de acordo com a 10a revisão da Classificação Internacional de Doenças (CID-10) 13. Assim, o caso aqui não representa um paciente, uma vez que um mesmo indivíduo pode ter mais de um câncer primário e, assim, representar mais de um caso na base. Além da identificação dos casos de câncer, é possível também identificar os casos novos em um determinado período. Um caso foi considerado novo quando a data de validade da sua primeira APAC pertencia ao período observado. 
Além dos campos disponíveis nos arquivos do módulo de Oncologia do APACSIA/SUS, foram criados os seguintes campos: caso novo, idade, grupo etário (criança ou adulto), intervalo de tempo entre a data de diagnóstico e a entrada no sistema, último mês em que o caso recebeu tratamento, meses em tratamento, valor do tratamento por caso, tratamento concluído e óbito. Foram ainda criadas seis tabelas auxiliares para o SISONCO, a saber:

- Municipios: código, nome e unidade da federação de todos os municípios do Brasil, de acordo com o Instituto Brasileiro de Geografia e Estatística (ftp://geoftp.ibge.gov.br/Organizacao/Divi sao_Territorial/2007/);

- Tipo_cancer: código e descrição dos tipos de câncer, segundo a CID-10 13;

- Unidades Prestadoras de Serviço (UPS): identificação, tipo de unidade, sendo Centro de Alta Complexidade em Oncologia (CACON) ou serviço isolado de quimioterapia ou radioterapia das UPS;

- Procedimentos: código, descrição, modalidade (quimioterapia e radioterapia), finalidade e linha dos procedimentos autorizados pelo APACSIA/SUS;

- Motivo_cobranca: código e a descrição dos motivos de cobrança de uma APAC.

Foram criadas duas estratégias, chamadas de manual e automática, para verificação da consistência da integração dos arquivos por meio do SISONCO. Para tal, utilizaram-se dois conjuntos de registros gerados pela rotina proposta por Gomes Jr. et al. 9. Para o primeiro conjunto, foram selecionados os registros de casos de câncer de estômago e pele não melanoma no período de janeiro a março de 2001. A escolha desses dois tipos de câncer deveu-se à relevância epidemiológica $14 \mathrm{e}$ à factibilidade da apuração manual. Desses registros, apuraram-se manualmente a quantidade de casos e casos novos, valor total apresentado e valor total aprovado. Na estratégia automática, foram utilizados os registros dos atendimentos efetuados para o ano de 2002, sendo computados: quantidade de casos, número de óbitos e valores apresentado e aprovado por caso.

Este projeto foi aprovado pelo Comitê de Ética em Pesquisa do Hospital Universitário Pedro Ernesto (CEP/HUPE - CAAe: 0153.0.228.000-07).

\section{Resultados}

O modelo do SISONCO possui duas tabelas principais, denominadas Casos e Historico. A tabela Casos contém os campos que descrevem as características de cada caso, sendo gerado um único registro por caso; a tabela Historico é com- posta por dados que descrevem a evolução do tratamento dos casos registrados mensalmente.

A tabela Casos ainda se relaciona com as tabelas auxiliares Entrada, Tipo_Cancer, Genero, Municipios e UPS. Dessa forma, é possível consultar os casos de câncer por diagnóstico principal, município de origem e unidade prestadora de serviço, na qual teve origem o primeiro tratamento do caso no módulo de oncologia do APACSIA/SUS (Figura 1).

A tabela Historico relaciona-se com a tabela Casos e com as tabelas auxiliares Tipo_Cancer, Municipios, UPS, Procedimentos e Motivo_cobranca, o que permite conhecer o histórico do tratamento do caso (Figura 1).

A interface gráfica do SISONCO apresenta no menu principal as opções Arquivos, Atualizacoes, Consultas e Ajuda. Em Arquivos, o usuário pode visualizar os tipos de câncer, procedimentos, municípios dos casos e UPS. A opção Atualizacoes permite ao usuário atualizar o sistema a partir dos arquivos do módulo de oncologia do APACSIA/SUS, obtidos no site do DATASUS (ftp://msbbs.datasus.gov.br/Arquivos_Publicos/ Estado_RJ/00_index.htm). Todo esse processo é realizado de forma automática, bastando ao usuário informar o ano e mês dos arquivos de produção.

O sistema oferece dois tipos de consulta: à lista de casos e sobre a produção de serviços. A consulta à lista de casos é feita de acordo com as opções do usuário para as variáveis: período da consulta, município de origem dos casos, unidade prestadora de serviço, tipo de unidade prestadora de serviço, tipo de câncer, modalidade terapêutica inicial, gênero, grupo etário dos casos, estadiamento do tumor e presença de metástase na entrada do caso no sistema. A Figura 2 mostra um exemplo de consulta para o período de janeiro a dezembro de 2001, dos casos de câncer de mama feminino, em adultos, originários do Município do Rio de Janeiro, que apresentavam metástase quando da entrada no sistema e que tiveram como primeiro tratamento um procedimento de radioterapia. O resultado dessa consulta pode ser exportado nos formatos: csv, xls e xml. Nessa tela, é ainda possível observar, de acordo com a opção escolhida, o número de casos identificados, a quantidade casos novos, a média de idade dos casos, o intervalo médio (em meses) entre as datas de diagnóstico e de entrada no sistema e o número óbitos. Selecionando um caso de interesse, é possível visualizar detalhes do tratamento ao se pressionar a tecla de Historico. A Figura 3 mostra algumas características gerais do caso: gênero, idade, tipo de câncer e início e valor do tratamento. Adicionalmente, são exibidos, para cada mês de tratamento, a 
Figura 1

Modelo do banco de dados do SISONCO.

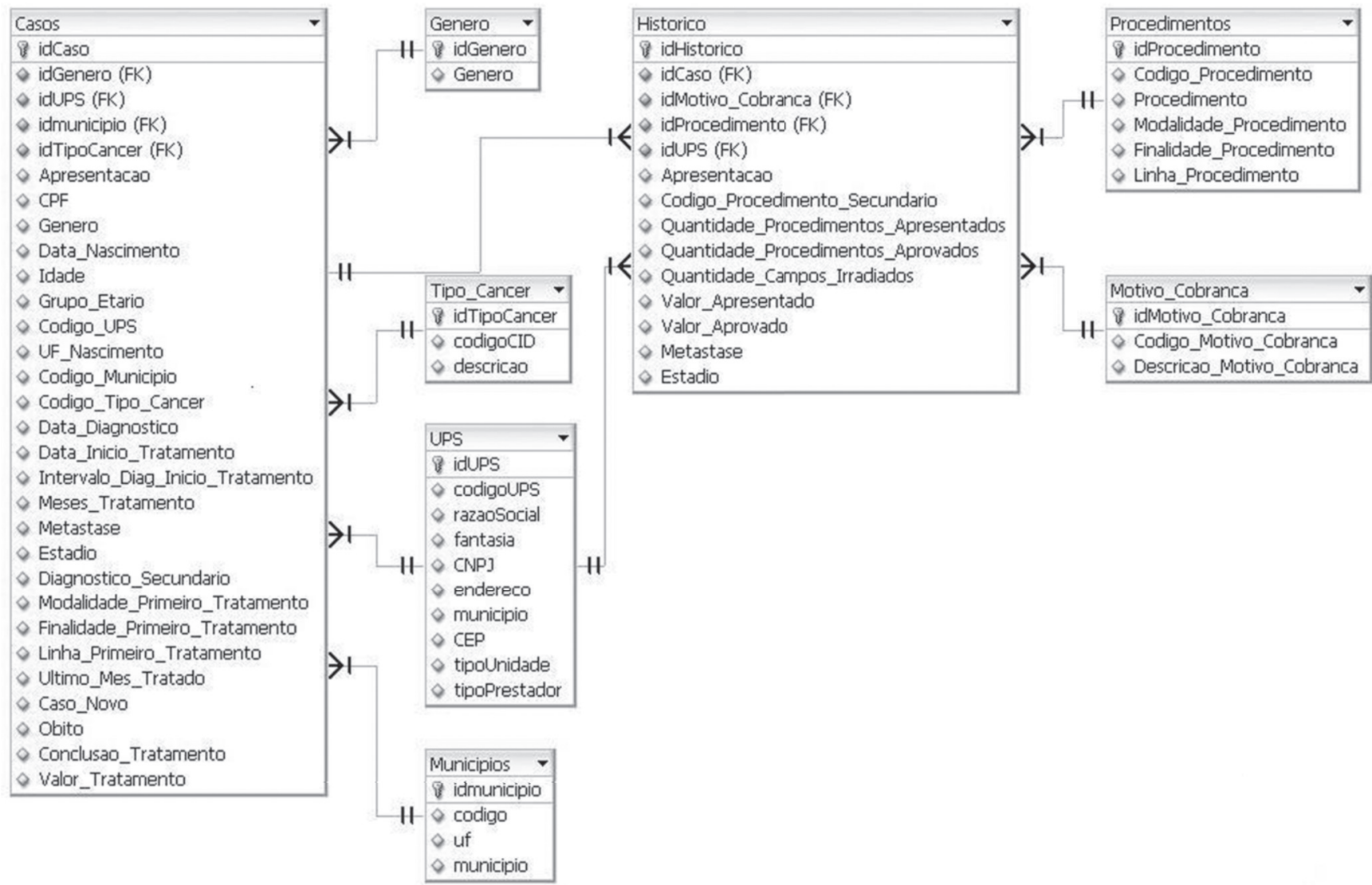

UPS onde se deu o atendimento, a modalidade terapêutica adotada, o valor aprovado, presença de metástase e estadiamento do tumor. Selecionando um mês de atendimento, é exibida uma segunda tabela com detalhes do tratamento: descrição do procedimento segundo a tabela de procedimentos da APAC, quantidade de campos irradiados (nos casos de radioterapia), valor apresentado e valor aprovado.

A tela de consulta sobre a produção de serviço é apresentada na Figura 4. Escolhe-se inicialmente a variável cujas categorias irão aparecer nas linhas da tabela de saída (tipo de câncer, UPS, tipo de UPS, município de localização da UPS ou modalidade terapêutica). Em seguida, selecionase o período de consulta na base e as categorias das variáveis que serão utilizadas como filtro na consulta. Os resultados dessa consulta são vistos na Figura 5, apresentando, para cada categoria da variável selecionada na linha, a quantidade de casos tratados, valor apresentado, valor aprovado, valor médio de tratamento por caso e média de campos irradiados.

Nos testes de avaliação das rotinas do SISONCO, utilizando a estratégia manual, foram encontrados 57 casos de câncer de estômago sendo 19 casos novos, representando valor total apresentado de $\mathrm{R} \$ 59.162,12$ e aprovado de R\$ 56.311,62 - e 34 casos de câncer de pele não melanoma - sendo 28 casos novos, representando um total apresentado de R\$ 12.397,06 e aprovado de $\mathrm{R} \$ 12.397,06$. Na estratégia automática, foram encontrados 18.384 casos de câncer com 700 óbitos no período; foi apresentado valor total de $\mathrm{R} \$ 54.834 .122,89$, sendo aprovados $\mathrm{R} \$ 54.707 .894,52$. Os resultados encontrados nas duas estratégias foram idênticos aos do SISONCO. 
Figura 2

Tela de consulta à lista de casos selecionados a partir das opções de filtragem.

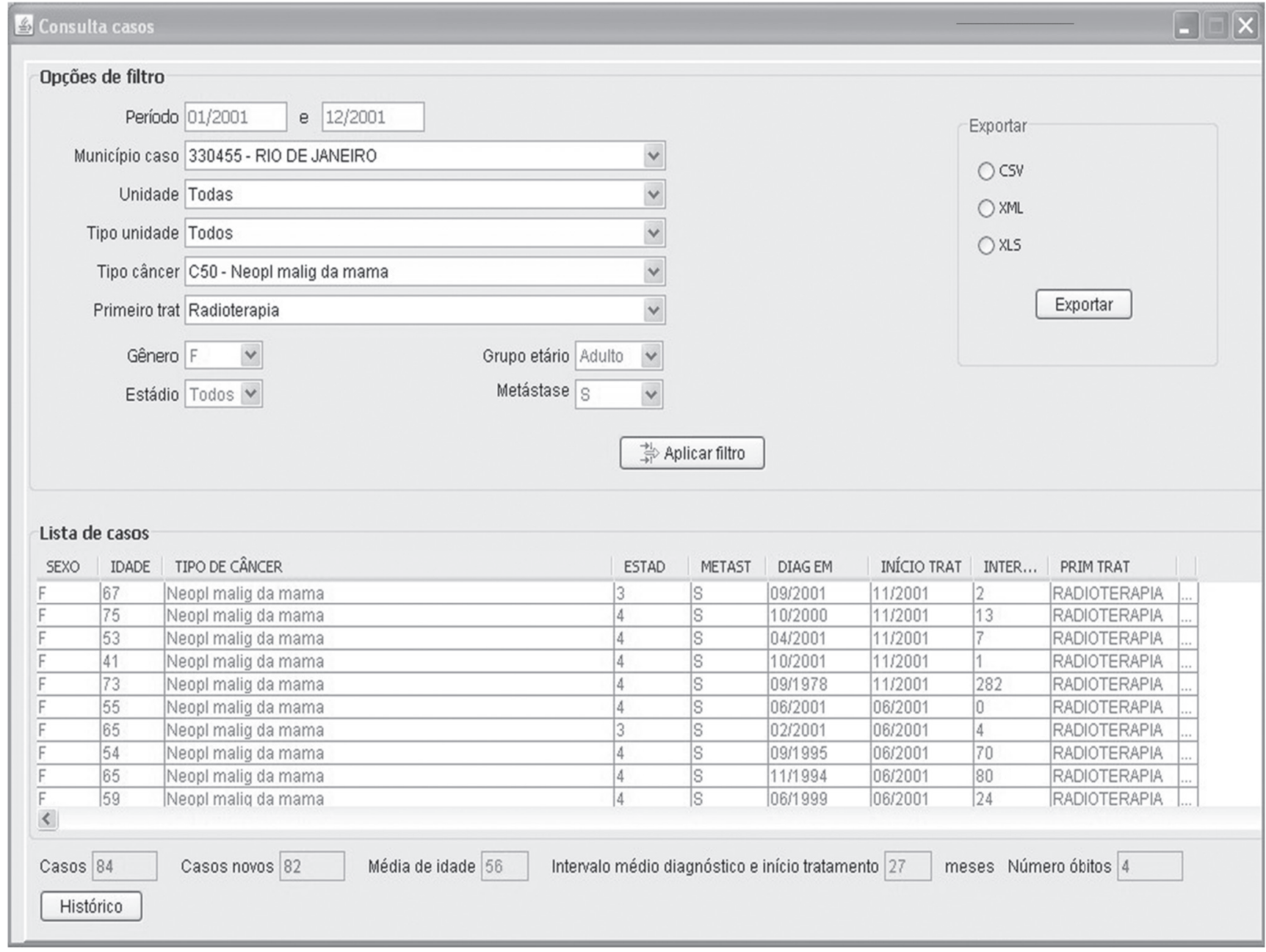

\section{Discussão}

Embora se reconheçam os esforços do Ministério da Saúde no desenvolvimento e implantação de sistemas de informação em saúde de abrangência nacional, ainda há desafios a serem vencidos visando à sua melhoria. Uma das limitações dos sistemas de informação desenvolvidos e mantidos pelo Ministério da Saúde é a não integração das diversas bases de dados. Entre sistemas de propósitos diferentes, o impedimento à vinculação se dá em virtude da ausência de uma chave que permita a identificação unívoca dos indivíduos. Mesmo quando se trabalha com um único sistema, como o APACSIA/SUS, a integração dos seus arquivos de dados pode depender de estratégia externa, como a rotina desenvolvida por Gomes Jr. et al. 9 .

O desenvolvimento do SISONCO surge como proposta de agregar à metodologia de Gomes Jr. et al. 9 facilidades operacionais para a integração dos arquivos, identificação de casos e geração de consultas, por meio de uma interface amigável. Quando comparado à estratégia de integração de Gomes Jr. et al. 9, o SISONCO apresenta as seguintes vantagens: (1) dispensa a necessidade de conhecimento de banco de dados para a construção de rotinas e aquisição de informações; (2) permite que a atualização do banco de dados seja feita de forma cumulativa, sem precisar 
Figura 3

Tela de visualização do histórico de tratamento de um caso selecionado.

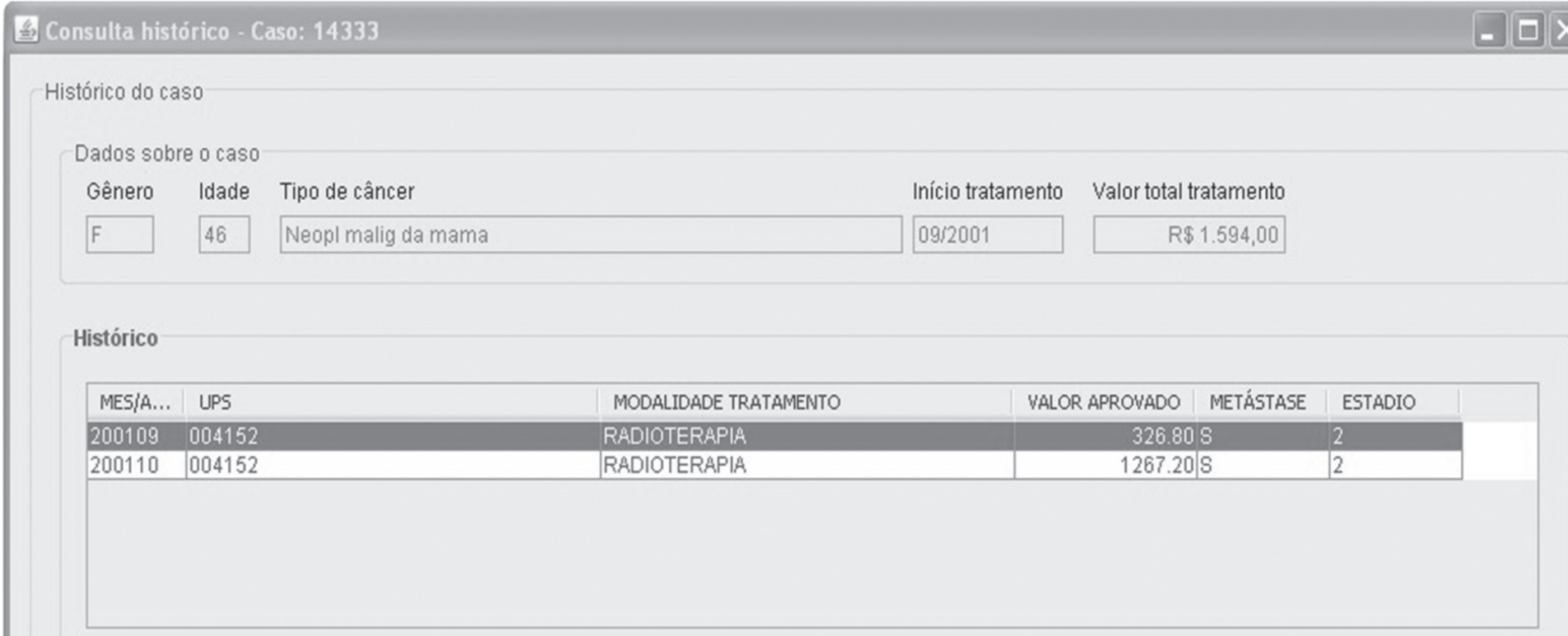

Detalhe tratamento

\begin{tabular}{|l|r|r|r|}
\hline DESCRIÇÄO PROCEDIMENTO APAC & CAMPOS IRRADIA... & VALOR APRESENT... & VALOR APROVADO \\
\hline PLANEJAMENTO SEM SIMULADOR (POR TRATAMENTO) & 0 & 10.00 & 10.00 \\
\hline COBALTOTERAPIA - POR CAMPO & 20 & 316.80 & \\
\hline & & \\
\end{tabular}

que todos os arquivos sejam integrados a cada atualização; (3) reduz o tempo de atualização e de extração da informação; (4) permite que as consultas sejam feitas a partir de uma interface gráfica.

No Brasil, existem experiências de desenvolvimento de sistemas que geram informações úteis para a gestão a partir das bases de produção dos sistemas implantados pelo DATASUS, destacando-se os trabalhos de Mussi et al. 15 e o de Santos et al. ${ }^{16}$. O SISONCO difere destes por permitir a identificação dos casos de câncer e a recuperação de seus históricos de tratamento no APACSIA/SUS, propiciando a análise longitudinal dos casos tratados.
Algumas dificuldades, identificadas nas bases originais do APACSIA/SUS, tiveram de ser tratadas durante o processo de criação do SISONCO. A forma como os dados são armazenados no APACSIA/SUS produz redundâncias, já que, a cada nova apresentação, os mesmos dados são fornecidos. Com isso, não há nesse sistema um cadastro único de casos tratados pelas unidades prestadoras de serviço. O SISONCO definiu tabela única de casos, de modo a permitir o cálculo da quantidade de casos tratados. Outro fato a chamar a atenção foi o aparecimento de inconsistências em alguns campos, como gênero e data de nascimento. Para exemplificar, em determinada UPS, observaram-se registros 


\section{Linha Municipio UPS}

Filtro

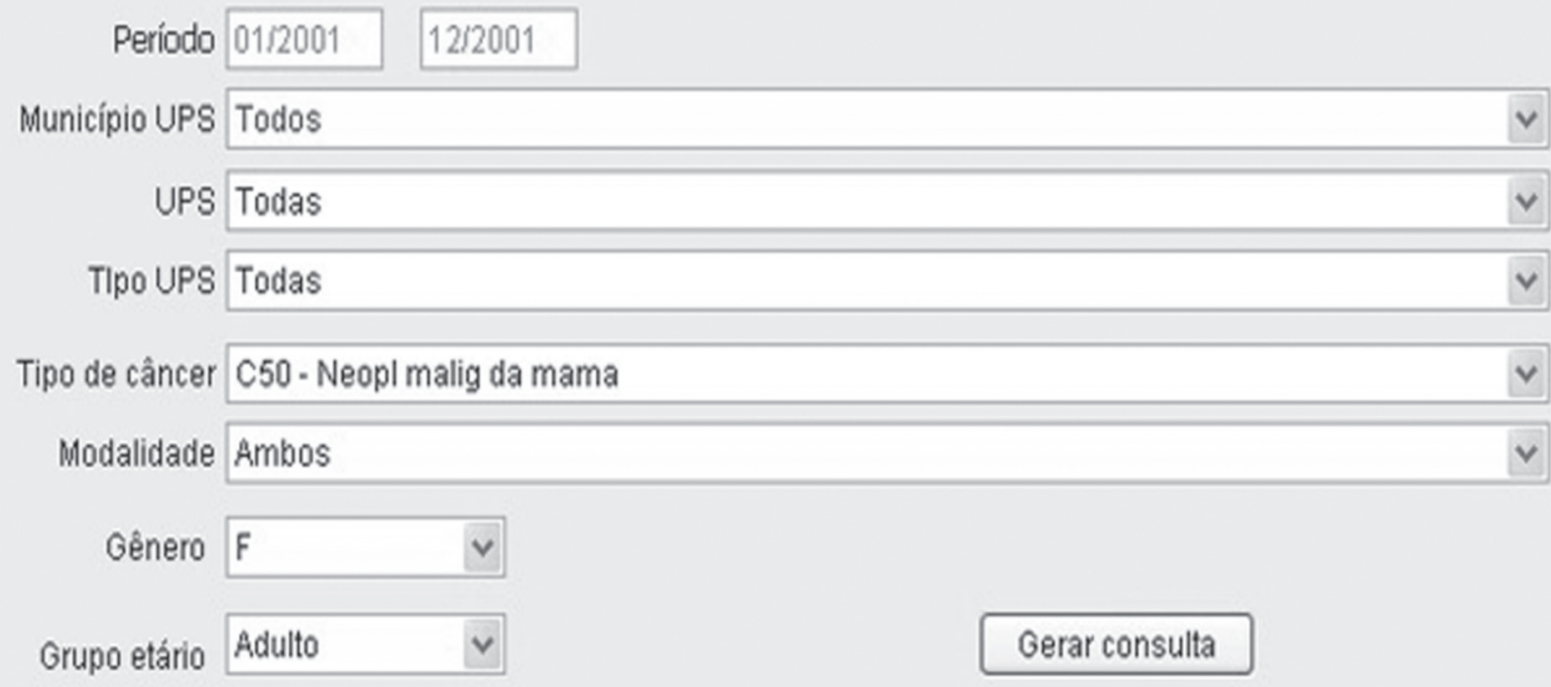

que apresentaram o mesmo CPF, gênero e tipo de câncer, mas que tiveram diferentes valores para a data de nascimento ao longo de um período. Como esses campos compõem a chave que indica um caso de câncer, tais inconsistências levam a considerar como diferentes um mesmo caso. Nessas situações, os casos foram computados mais de uma vez.

Algumas características não disponíveis na atual versão precisam ser incorporadas ao SISONCO, destacando-se as seguintes:

- Tratamento para atualização das tabelas auxiliares. Um exemplo de necessidade desse tipo de tratamento é o das unidades prestadoras de serviços que, até 2002, recebiam um tipo de codificação e, a partir de 2003, passaram a ser codificadas pelo Cadastrado Nacional de Estabelecimentos de Saúde (CNES). Nessa situação, o sistema precisa reconhecer os registros com os tipos de códigos diferentes como pertencentes a uma mesma UPS.

- Integração da base de dados do SISONCO a ferramentas para análises de dados, conhecidas como On Line Analytical Processing (OLAP). Essas ferramentas facilitam a construção de consultas analíticas e a navegação nas informações resultantes, possibilitam a geração de relatórios, apresentação de resultados em forma de gráficos e a mineração de dados. 
Figura 5

Tela de visualização dos dados de produção.

붕. Resultados da consulta sobre a produçao

- $\square x$

\begin{tabular}{|c|c|c|c|c|c|}
\hline Variável & Casos & Valor apresentado & Valor aprovado & Valor médio tratamento & Média campos irradiados \\
\hline RIO DE JANEIRO & 5897 & $14.760 .017,09$ & $14.226 .626,82$ & $2.412,52$ & 31 \\
\hline CAMPOS DOS GOYTACAZES & 435 & $1.119,452,16$ & $1.119,452,16$ & $2.573,45$ & 26 \\
\hline NOVA IGLACU & 393 & $891.453,18$ & $878.327,91$ & $2.234,93$ & 53 \\
\hline VOLTÁ REDONDÁ & 350 & $889,992,05$ & $886.405,13$ & $2.532,59$ & 30 \\
\hline PETROPOLIS & 323 & $828.829,35$ & $822.756,65$ & $2.547,23$ & 40 \\
\hline MESQQUITA & 106 & $826.546,40$ & $826.546,40$ & $7,797,61$ & 0 \\
\hline ITAPERUNNA & 97 & $156,089,78$ & $149,020,80$ & $1.536,30$ & 105 \\
\hline NITEROI & 92 & $164.774,32$ & $164.731,26$ & $1.790,56$ & 41 \\
\hline TERESOPOLIS & 65 & $229.518,15$ & $229.518,15$ & $3.531,05$ & 0 \\
\hline MAGE & 20 & $31.139,50$ & $31.139,50$ & $1.556,98$ & 0 \\
\hline
\end{tabular}

Nessa primeira etapa, o SISONCO foi apenas avaliado em relação à correção dos resultados obtidos. Em uma segunda fase do projeto, espera-se avaliar o sistema na percepção dos usuários, tanto no tocante às consultas que ele oferece, quanto à facilidade de sua utilização.
Finalmente, o desenvolvimento do SISONCO está sendo orientado pela lógica de software livre. Portanto, após a conclusão da sua primeira versão, ele estará disponível ao público em geral para acesso e uso. Espera-se que ferramentas como esta possam contribuir para a melhoria da gestão da assistência oncológica ambulatorial prestada pelo SUS. 


\section{Resumo}

Este trabalho visa ao desenvolvimento de um sistema para integrar os dados da produção mensal da assistência oncológica ambulatorial no Sistema Único de Saúde (SUS). O sistema foi modelado para recuperar a evolução do tratamento de cada caso de câncer e a produção dos serviços, tendo como vantagens: ser orientado ao caso de câncer, não requerer do usuário conhecimentos para a manipulação de bancos de dados e permitir a atualização da base à medida que novos dados vão surgindo. Os resultados das consultas ao sistema, quando comparados com os obtidos na inspeção direta da base original e com os obtidos com outra rotina de integração, foram idênticos. A utilização de uma ferramenta com essas características pelos gestores públicos pode contribuir para a melhoria da gestão da assistência oncológica ambulatorial prestada pelo SUS.

Sistemas de Informação em Atendimento Ambulatorial; Assistência Ambulatorial; Neoplasias

\section{Referências}

1. Moura L, Curado MP, Simões EJ, Cezário AC, Urdaneta M. Avaliação do Registro de Câncer de Base Populacional do Município de Goiânia, Estado de Goiás, Brasil. Epidemiol Serv Saúde 2006; 15:7-17.

2. Brasil. Portaria ${ }^{\circ}$. 3.536, de 2 de setembro de 1998. Dispõe sobre a implantação dos formulários e regulamenta sua utilização na sistemática de autorização e cobrança dos procedimentos ambulatoriais na área do câncer. Diário Oficial da União 1998; 14 out

3. Ministério da Saúde. Informativo SUS-ONCO 1999; Ano 4, n. 07. http://dtr2001.saude.gov.br/sas/de cas/onconov99.htm (acessado em 11/Jun/2005).

4. Bittencourt R, Scaletzky A, Rossi JA. Perfil epidemiológico do câncer na rede pública em Porto Alegre-RS. Rev Bras Cancerol 2004; 50:95-101.

\section{Colaboradores}

R. C. Souza participou da concepção, modelagem, implementação, coleta dos dados e redação do artigo. S. M. Freire e R. T. Almeida colaboraram na concepção, modelagem e redação do artigo.
5. Gadelha PMI, Costa MR, Almeida RT. Estadiamento de tumores malignos: análise e sugestões a partir de dados da APAC. Rev Bras Cancerol 2005; 51:193-9.

6. Brito C, Portela MC, Vasconcellos MTL. Assistência oncológica pelo SUS a mulheres com câncer de mama no Estado do Rio de Janeiro. Rev Saúde Pública 2005; 39:874-81.

7. Costa MR. Comparação das condutas terapêuticas no tratamento ambulatorial das mulheres com câncer de mama [Dissertação de Mestrado]. Rio de Janeiro: Instituto Alberto Luiz Coimbra de Pósgraduação em Engenharia, Universidade Federal do Rio de Janeiro; 2005. 
8. Gomes Jr. SCS, Almeida RT. Modelo de simulação para estimar a infraestrutura necessária à assistência oncológica no sistema público de saúde. Rev Panam Salud Pública 2009; 25:113-9.

9. Gomes Jr. SCS, De Martino R, Almeida RT. Rotinas de integração das tabelas do Sistema de Autorização de Procedimentos de Alta Complexidade em Oncologia do Sistema Único de Saúde. Cad Saúde Colet (Rio J.) 2003; 11:231-54.

10. Date, CJ. Introdução a sistemas de bancos de dados. 8a Ed. Rio de Janeiro: Editora Campus; 2004.

11. Miles R, Hamilton K. Learning UML 2.0. Sebastopol: O'Reilly Media Inc.; 2006.

12. Gomes Jr. SCS, Almeida RT. Identificação de um caso novo de câncer no Sistema de Informação Ambulatorial do Sistema Único de Saúde. Cad Saúde Colet (Rio J.) 2004; 12:57-68.
13. Organização Mundial da Saúde. Classificação estatística internacional de doenças e problemas relacionados à saúde, 10a revisão. v. 1. São Paulo: Universidade de São Paulo; 2007.

14. Instituto Nacional de Câncer. Estimativa 2008: incidência do câncer no Brasil. http://www.inca.gov. br/estimativa/2008 (acessado em 04/Fev/2008).

15. Mussi C, Murahovschi D, Bettini G, Kratz LG. Data warehouse: a experiência da ANVISA. In: Anais do IX Congresso Brasileiro de Informática em Saúde http://www.anvisa.gov.br/divulga/eventos/cbis_ mussi.pdf (acessado em Set/2009).

16. Santos RS, Gutierrez MA. MINERSUS - ambiente computacional para extração de informações para a gestão da saúde pública por meio da mineração dos dados do SUS. Rev Bras Eng Biomed 2008; 24:77-90.

Recebido em 23/Out/2009

Versão final reapresentada em 04/Fev/2010 Aprovado em 05/Abr/2010 